PROCEEDINGS OF THE

AMERICAN MATHEMATICAL SOCIETY

Volume 138, Number 6, June 2010, Pages 2245-2252

S 0002-9939(10)10246-9

Article electronically published on February 1, 2010

\title{
RESOLVABLE MAPS PRESERVE COMPLETE METRIZABILITY
}

\author{
SU GAO AND VINCENT KIEFTENBELD
}

(Communicated by Julia Knight)

\begin{abstract}
Let $X$ be a Polish space, let $Y$ be a separable metrizable space, and let $f: X \rightarrow Y$ be a continuous surjection. We prove that if the image under $f$ of every open set or every closed set is resolvable, then $Y$ is Polish. This generalizes similar results by Sierpiński, Vainštain, and Ostrovsky.
\end{abstract}

\section{INTRODUCTION}

Polish spaces (separable, completely metrizable spaces) are abundant in mathematics. Familiar examples are separable Banach spaces, the Baire space $\omega^{\omega}$, and the Cantor space $2^{\omega}$. These spaces are the natural setting for descriptive set theory. It is therefore of interest to find criteria when a separable metrizable space is in fact completely metrizable.

Consider a continuous surjection $f: X \rightarrow Y$ between separable metrizable spaces $X$ and $Y$. We say that $f$ preserves complete metrizability if $Y$ is completely metrizable whenever $X$ is completely metrizable. A natural question to ask is which maps preserve complete metrizability. Obviously, some restrictions need to be imposed on the map, since there are continuous maps from $\omega^{\omega}$ onto $\mathbb{Q}$. Moreover, Michael and Stone [7] proved that if there is a continuous map from $\omega^{\omega}$ onto a metrizable space $X$, then there is a quotient map from $\omega^{\omega}$ onto $X$. Thus quotient maps do not preserve complete metrizability.

A classical theorem of Sierpiński 9] (cf. also Hausdorff [2]) showed that open maps preserve complete metrizability. Later Vainštain [10] proved that closed maps preserve complete metrizability. There has been much work on other kinds of maps since then. Very recently, Ostrovsky 8 obtained the following result: if the image of every open set or every closed set is the union of an open and a closed set, then the map preserves complete metrizability. He raised the question whether the same is true when the images are the intersection of an open set and a closed set.

An intersection of an open set and a closed set can also be written as the difference of two open sets. Indeed, these sets form the second level of the difference hierarchy introduced by Hausdorff. More complicated combinations of open and closed sets occur at higher levels of this hierarchy, and all sets in the difference hierarchy are known as resolvable sets. A detailed account of the structure of the

Received by the editors July 15, 2009, and, in revised form, October 5, 2009.

2010 Mathematics Subject Classification. Primary 54E40, 54E50; Secondary 03E15, 54H05.

Key words and phrases. Complete metrizability, resolvable sets.

The first author acknowledges the support of NSF grants DMS-0501039 and DMS-0901853.

The second author acknowledges the support of NSF grant DMS-0901853. 
difference hierarchy can be found, e.g., in [5, Section 22.E]. Hausdorff and Kuratowski (cf. [5, Theorem 22.27]) showed that in a Polish space the resolvable sets are exactly the $\boldsymbol{\Delta}_{2}^{0}$ sets, i.e., sets that are both $F_{\sigma}$ and $G_{\delta}$. However, for general separable metrizable spaces this characterization fails. There, every resolvable set is $\boldsymbol{\Delta}_{2}^{0}$, but the converse does not hold (cf. Proposition 4 below).

Let $X$ and $Y$ be separable metrizable spaces. We say that a map $f: X \rightarrow Y$ is open-resolvable if the image under $f$ of every open set is resolvable. Similarly, we say that $f$ is closed-resolvable (clopen-resolvable) if the image under $f$ of every closed (clopen) set is resolvable. Finally, we say that $f$ is resolvable if it is either open-resolvable or closed-resolvable. We will prove the following generalization of Ostrovsky's theorem.

Theorem 1. If $X$ is Polish, $Y$ is metrizable, and $f: X \rightarrow Y$ is a resolvable continuous surjection, then $Y$ is Polish.

This answers Ostrovsky's question in the affirmative. We remark that this seems to be the strongest generalization one can obtain along this line, again because there is a continuous map from $\omega^{\omega}$ onto $\mathbb{Q}$. Every subset of $\mathbb{Q}$ is $F_{\sigma}$ and hence $\boldsymbol{\Delta}_{2}^{0}$, and therefore our theorem cannot be further strengthened by replacing resolvable maps by maps sending open (or closed) sets to $\boldsymbol{\Delta}_{2}^{0}$ sets.

A somewhat related question is whether the difference hierarchy collapses for certain separable metrizable spaces. We can prove that for any separable metrizable space whose completion is uncountable the difference hierarchy does not collapse. This confirms that our theorem is a genuine generalization of Ostrovsky's theorem.

In addition, we note that not every map which preserves complete metrizability is necessarily resolvable. Consider any Polish space $(X, \sigma)$ and any Borel set $B \subseteq X$ which is not resolvable, that is, $B \notin \boldsymbol{\Delta}_{2}^{0}$. There is a finer Polish topology $\tau \supseteq \sigma$ on $X$ such that $B$ is clopen in $\tau$ [5, Theorem 13.1]. The identity map between $(X, \tau)$ and $(X, \sigma)$ is a continuous surjection between completely metrizable spaces but is not resolvable.

The rest of the paper is organized as follows. In Section 2 we prove some preliminary results needed in later proofs. In particular, we show that the range of a continuous resolvable map is absolute Borel and give some characterizations of nonresolvable subsets of $\mathbb{Q}$. Then we show in Section 3 that there cannot be a clopen-resolvable map $f: \omega^{\omega} \rightarrow \mathbb{Q}$, and from this we derive Theorem 1 in Section 4 .

While preparing this paper for publication, we obtained a preprint by Holický and Pol [3], who independently proved the same result. We thank them for discussions on the subject.

\section{Preliminaries}

We first show that the range of a continuous resolvable map is absolute Borel.

Recall that a metrizable space $X$ is said to be absolute Borel if for any metrizable space $Y$ and homeomorphic embedding $j: X \rightarrow Y, j(X)$ is a Borel subset of $Y$. The following useful criterion for absolute Borelness for continuous images of Polish spaces is well-known (see e.g., [8]).

Proposition 2. Let $X$ be a Polish space, let $Y$ be a separable metrizable space, and let $f: X \rightarrow Y$ be a continuous surjection. If the image under $f$ of every open set in $X$ is Borel in $Y$, then $Y$ is absolute Borel. 
Proof. Let $E$ be the equivalence relation on $X$ defined by $x_{1} E x_{2}$ iff $f\left(x_{1}\right)=$ $f\left(x_{2}\right)$. By the continuity of $f$ every $E$-equivalence class is closed. The condition is equivalent to the statement that the $E$-saturation of every open set is Borel, since $[U]_{E}=f^{-1}(f(U))$. We consider the map $\theta: X \rightarrow F^{*}(X)$, where $F^{*}(X)$ is the standard Borel space of all nonempty closed subsets of $X$ with the Effros Borel structure (c.f. [5, Section 12.C]), defined by $\theta(x)=[x]_{E}=f^{-1}(f(x))$. Then $\theta$ is Borel since for any nonempty open $U \subseteq X$,

$$
\theta(x) \cap U \neq \emptyset \Longleftrightarrow x \in[U]_{E} .
$$

Let $\sigma: F^{*}(X) \rightarrow X$ be the Borel selector function given by the Kuratowski-RyllNardzewski theorem (see [5, Theorem 12.13]). Then $\sigma \circ \theta: X \rightarrow X$ is a Borel selector for $E$. Let $A=\sigma \circ \theta(X)$. Then $A$ is a Borel transversal for $E$; it is Borel since $x \in A$ iff $\sigma \circ \theta(x)=x$. Thus $A$ is a separable absolute Borel space and $f\lceil A: A \rightarrow Y$ is a continuous bijection. It follows from a classical theorem of Luzin-Suslin ([5, Theorem 15.1]) that $Y$ is absolute Borel.

In particular, in the above proposition if $f$ is open-resolvable, then $Y$ is absolute Borel. To show the similar result for closed-resolvable maps, we use the following result of Engelking [1]: for every Polish space $X$ there is a closed continuous map from $\omega^{\omega}$ onto $X$.

Proposition 3. Let $X$ be a Polish space, let $Y$ be a separable metrizable space, and let $f: X \rightarrow Y$ be a continuous surjection. If the image under $f$ of every closed set in $X$ is Borel in $Y$, then $Y$ is absolute Borel.

Proof. Let $g: \omega^{\omega} \rightarrow X$ be a closed continuous map from $\omega^{\omega}$ onto $X$. Then $f \circ$ $g: \omega^{\omega} \rightarrow Y$ is continuous, and the image under $f \circ g$ of every clopen set in $\omega^{\omega}$ is Borel in $Y$. This implies that the image under $f \circ g$ of every open set in $\omega^{\omega}$ is Borel in $Y$, since every open set in $\omega^{\omega}$ is a countable union of clopen sets. Now applying Proposition 2 to $f \circ g$, we conclude that $Y$ is absolute Borel.

We have thus shown that the range of a continuous resolvable map is absolute Borel. It is a theorem of Hurewicz [4 that a separable metrizable, coanalytic (in particular, absolute Borel) space $Y$ is completely metrizable if and only if $Y$ does not contain a countable perfect set $Q \subseteq Y$ (see [5, Theorem 21.18]). Fréchet and Sierpiński (5. Exercise 7.12]) have proved that such a countable perfect set must be homeomorphic to $\mathbb{Q}$.

Next we turn to nonresolvable subsets of $\mathbb{Q}$. As we noted, every subset of $\mathbb{Q}$ is $\boldsymbol{\Delta}_{2}^{0}$, but not every subset of $\mathbb{Q}$ is resolvable. We will use the following characterizations of nonresolvable subsets of $\mathbb{Q}$.

Proposition 4. Let $A \subseteq \mathbb{Q}$. The following are equivalent:

(1) $A$ is not resolvable.

(2) $A$ is not relatively $\boldsymbol{\Delta}_{2}^{0}$; i.e., there is no $\boldsymbol{\Delta}_{2}^{0}$ subset $B$ of $\mathbb{R}$ such that $B \cap \mathbb{Q}=$ $A$.

(3) $A$ is dense, codense in a closed $F \subseteq \mathbb{Q}$.

(4) $A$ is dense, codense in a perfect $F \subseteq \mathbb{Q}$.

(5) $A$ is dense, codense in a homeomorphic copy of $\mathbb{Q}($ inside $\mathbb{Q}$ ).

Proof. The equivalence of (1) and (2) follows from [5, Exercise 22.26]. In fact, if $A$ is resolvable in $\mathbb{Q}$, then its representation in the difference hierarchy of $\mathbb{Q}$ can be lifted to the difference hierarchy of $\mathbb{R}$ to obtain a set $B \subseteq \mathbb{R}$ resolvable in $\mathbb{R}$ so that 
$B \cap \mathbb{Q}=A$. Since $B$ is $\boldsymbol{\Delta}_{2}^{0}, A$ is relatively $\boldsymbol{\Delta}_{2}^{0}$. Conversely, if $B \subseteq \mathbb{R}$ is $\boldsymbol{\Delta}_{2}^{0}$ and $B \cap \mathbb{Q}=A$, then $B$ is resolvable, and the restriction to $\mathbb{Q}$ of its representation in the difference hierarchy of $\mathbb{R}$ gives a representation of $A$ in the difference hierarchy of $\mathbb{Q}$.

$(1) \Rightarrow(3)$ is a consequence of the proof of the Hausdorff-Kuratowski theorem 5 , Theorem 22.27]. In fact, the standard proof of this theorem utilizes a transfinite recursion to define a decreasing sequence $\left(F_{\alpha}\right)_{\alpha<\omega_{1}}$ of closed sets in which $A$ is dense. Let $\theta<\omega_{1}$ be the least number such that $F_{\theta}=F_{\theta+1}$. The proof shows that if $F_{\theta}=\emptyset$, then $A$ is resolvable, and otherwise $A$ is dense, codense in $F_{\theta}$.

$(3) \Rightarrow(4)$ Assume $A$ is dense, codense in a closed set $F \subseteq \mathbb{Q}$. Suppose $x \in F$ is an isolated point. Then $\{x\}$ is open in $F$; hence $x \in A$ because $A$ is dense. Similarly, $x \in F \backslash A$ because $A$ is codense. This is a contradiction.

$(4) \Rightarrow(5)$ If $F \subseteq \mathbb{Q}$ is perfect, then $F$ is homeomorphic to $\mathbb{Q}$ by the FréchetSierpiński result (see [5, Exercise 7.12]).

$(5) \Rightarrow(2)$ We may assume that $A$ is dense, codense in $\mathbb{Q}$ itself. Suppose $A=B \cap \mathbb{Q}$ where $B$ is a $\Delta_{2}^{0}$ subset of $\mathbb{R}$. Then $B$ and $\mathbb{R} \backslash B$ are both dense $G_{\delta}$ in $\mathbb{R}$. But $B$ and $\mathbb{R} \backslash B$ are disjoint, a contradiction to the fact that $\mathbb{R}$ is a Baire space.

\section{Continuous surjections from $\omega^{\omega}$ Onto $\mathbb{Q}$}

In this section we show that there is no clopen-resolvable map from a closed subset $P \subseteq \omega^{\omega}$ onto $\mathbb{Q}$.

For any $s=\left(s_{0}, \ldots, s_{k}\right) \in \omega^{<\omega}$ we denote by $N_{s}$ the set $\left\{x \in \omega^{\omega}: s \subseteq\right.$ $x\}$. If $n \in \omega$, then we denote by $s^{\frown} n$ the sequence $\left(s_{0}, \ldots, s_{k}, n\right)$. Recall that a tree on $\omega$ is a set $T \subseteq \omega^{<\omega}$ of finite sequences of natural numbers such that if $\left(t_{0}, \ldots, t_{n}\right) \in T$, then $\left(t_{0}, \ldots, t_{m}\right) \in T$ for all $m \leq n$. The body of a tree $T$ is the set $[T]=\left\{x \in \omega^{\omega}: \forall n \in \omega(x\lceil n \in T)\}\right.$. A tree $T$ is pruned if $N_{s} \cap[T] \neq \emptyset$ for any $s \in T$. When $P \subseteq \omega^{\omega}$ is closed, there is a unique pruned tree $T$ on $\omega$ such that $P=[T]$ ([5, Sections 2.A and 2.B]). For notational simplicity, when $P \subseteq \omega^{\omega}$ and $f: P \rightarrow \mathbb{Q}$, we write $f\left(N_{s}\right)$ for $f\left(N_{s} \cap P\right)$.

Lemma 5. Let $T$ be a pruned tree on $\omega$, let $P=[T]$, and let $f: P \rightarrow \mathbb{Q}$ be a continuous surjection. Suppose there is an $s \in T$ and a nonempty open set $O \subseteq \mathbb{Q}$ such that

(1) $f\left(N_{s^{\frown} n}\right)$ is nowhere dense in $O$ for all $n \in \omega$, and

(2) $f\left(N_{s}\right)=\bigcup_{n \in \omega} f\left(N_{s^{\urcorner} n}\right)$ is dense in $O$.

Then there is a clopen $U \subseteq P$ such that $f(U)$ is not resolvable.

Proof. First, note that for every cofinite $S \subseteq \omega, \bigcup_{n \in S} f\left(N_{s^{\wedge} n}\right)$ is dense in $O$. To see this, suppose $\omega \backslash S=\left\{n_{0}, \ldots, n_{k}\right\}$ and $B \subseteq O$ is basic open. Since $f\left(N_{\mathcal{S}^{\wedge} n_{0}}\right)$ is nowhere dense in $O$, there is a nonempty open $B_{0} \subseteq B$ such that $f\left(N_{s>n_{0}}\right) \cap B_{0}=\emptyset$. Similarly, there is a nonempty open $B_{1} \subseteq B_{0}$ such that $f\left(N_{s\urcorner n_{1}}\right) \cap B_{1}=\emptyset$, etc. We thus get a nonempty open $B_{k} \subseteq O$ such that for all $i=0, \ldots, k, f\left(N_{s^{\wedge} n_{i}}\right) \cap B_{k}=\emptyset$. Since $\bigcup_{n \in \omega} f\left(N_{s^{\frown} n}\right)$ is dense in $O$, for some $n \in \omega, f\left(N_{s^{\wedge} n}\right) \cap B_{k} \neq \emptyset$. Hence, $n \in S$.

To prove the lemma it suffices to define a clopen $U \subseteq P$ such that $f(U)$ is dense, codense in $O$. Since $O$ is homeomorphic to $\mathbb{Q}, f(U)$ will then be nonresolvable by Proposition 4. We will recursively define a sequence $N_{n}, n \in \omega$, of basic clopen sets and take $U=\bigcup_{n} N_{n}$.

Enumerate all basic open sets contained in $O$ as $B_{0}, B_{1}, \ldots$ Let $\operatorname{lh}(s)=k$ be the length of $s$. At stage 0 , pick $x_{0}, y_{0} \supseteq s$ such that $f\left(x_{0}\right), f\left(y_{0}\right) \in B_{0}$ and 
$f\left(x_{0}\right) \neq f\left(y_{0}\right)$. Let $p_{0}=x_{0}(k)$ and $q_{0}=y_{0}(k)$. Then let $N_{0} \subseteq N_{s \frown p_{0}}$ be a basic clopen neighborhood of $x_{0}$ such that $f\left(y_{0}\right) \notin f\left(N_{0}\right)$. It is possible to pick such an $N_{0}$ since $f$ is continuous. Note that $f\left(N_{0}\right) \subseteq f\left(N_{s>p_{0}}\right)$ is nowhere dense in $O$. Also by the claim in the first paragraph of this proof, the set $\bigcup_{m \neq p_{0}, q_{0}} f\left(N_{s^{\urcorner} m}\right)$ is still dense in $O$.

At stage 1 we first pick an $x_{1} \supseteq s$ such that $x_{1}(k) \neq p_{0}, q_{0}$ and $f\left(x_{1}\right) \in B_{1}-$ $\left\{f\left(x_{0}\right), f\left(y_{0}\right)\right\}$. Next pick $y_{1} \supseteq s$ such that $y_{1}(k) \neq p_{0}, q_{0}$ and $f\left(y_{1}\right) \in B_{1}-$ $f\left(N_{0}\right)-\left\{f\left(x_{1}\right)\right\}$. Let $p_{1}=x_{1}(k)$ and $q_{1}=y_{1}(k)$. Then let $N_{1} \subseteq N_{s^{\frown}} p_{1}$ be a basic clopen neighborhood of $x_{1}$ such that $f\left(y_{0}\right), f\left(y_{1}\right) \notin f\left(N_{1}\right)$. Now $f\left(y_{0}\right), f\left(y_{1}\right) \notin$ $f\left(N_{0}\right) \cup f\left(N_{1}\right)$ and $f\left(N_{0}\right) \cup f\left(N_{1}\right)$ is still nowhere dense in $O$. Again the set $\bigcup_{m \neq p_{0}, q_{0}, p_{1}, q_{1}} f\left(N_{s\urcorner m}\right)$ is still dense in $O$.

In general, at stage $n$, pick $x_{n} \supseteq s$ such that $x_{n}(k) \neq x_{i}(k), y_{i}(k)$ for all $0 \leq i<n$ and $f\left(x_{n}\right) \in B_{n}-\left\{f\left(x_{i}\right), f\left(y_{i}\right): 0 \leq i<n\right\}$. Pick $y_{n} \supseteq s$ such that $y_{n}(k) \neq$ $x_{i}(k), y_{i}(k)$ for all $0 \leq i<n$ and $f\left(y_{n}\right) \in B_{n}-\bigcup_{0 \leq i<n} f\left(N_{i}\right)-\left\{f\left(x_{n}\right)\right\}$. Then let $N_{n} \subseteq N_{s^{\frown} x_{n}(k)}$ be a basic clopen neighborhood of $x_{n}$ such that $f\left(y_{i}\right) \notin f\left(N_{n}\right)$ for all $0 \leq i<n$. We actually have $f\left(y_{i}\right) \notin f\left(N_{0}\right) \cup \cdots \cup f\left(N_{n}\right)$ for $0 \leq i \leq n$ and that $f\left(N_{0}\right) \cup \cdots \cup f\left(N_{n}\right)$ is nowhere dense in $O$.

This finishes the definition of $N_{n}$ for $n \in \omega$, and we let $U=\bigcup_{n \in \omega} N_{n}$. Then $U$ is clearly clopen by the construction. Also, $f(U)$ is dense in $O$, since $f\left(x_{n}\right) \in f(U)$ for each $n \in \omega$, and $f\left(x_{n}\right) \in B_{n}$. Similarly, $f(U)$ is codense in $O$, since $f\left(y_{n}\right) \notin f(U)$ but $f\left(y_{n}\right) \in B_{n}$.

The following lemma is more general, but the proof is the same as above. We state it without proof.

Lemma 6. Let $T$ be a pruned tree, let $P=[T]$, and let $f: P \rightarrow \mathbb{Q}$ be a continuous surjection. Suppose there are $s, t_{n} \in T, n \in \omega$, and a nonempty open set $O \subseteq \mathbb{Q}$ such that

(1) $s \subseteq t_{n}$ for all $n \in \omega, N_{t_{n}} \cap N_{t_{m}}=\emptyset$ for $n \neq m$, and $N_{s} \cap P=\bigcup_{n} N_{t_{n}} \cap P$;

(2) $f\left(N_{t_{n}}\right)$ is nowhere dense in $O$ for all $n \in \omega$; and

(3) $f\left(N_{s}\right)=\bigcup_{n} f\left(N_{t_{n}}\right)$ is dense in $O$.

Then there is a clopen $U \subseteq P$ such that $f(U)$ is not resolvable.

Proposition 7. Let $P \subseteq \omega^{\omega}$ be closed and let $f: P \rightarrow \mathbb{Q}$ be a continuous surjection. Then there is a clopen $U \subseteq P$ such that $f(U)$ is not resolvable.

Proof. Let $T$ be the unique pruned tree with $P=[T]$. We describe a search algorithm to find $s, t_{n} \in T, n \in \omega$, and $O \subseteq \mathbb{Q}$ satisfying the assumptions of Lemma 6. The search will be conducted by induction on the lengths of the nodes in $T$ and will produce a sequence $T \supseteq T_{0} \supseteq T_{1} \supseteq \ldots$ of subtrees of $T$, where some of the nodes are labeled with nonempty open subsets of $\mathbb{Q}$. We will maintain the following properties for all $l \in \omega$ :

(i) $T_{l} \subseteq T$ contains all labeled nodes;

(ii) $\left[T_{l}\right]$ is clopen in $[T]$;

(iii) if $s \in T_{l}$ is labeled with $O$ at stage $l$, then $f\left(N_{s} \cap\left[T_{l}\right]\right)$ is dense in $O$; and

(iv) if $s_{1} \subsetneq s_{2}$ first obtain labels at stages $l_{1}, l_{2}$ respectively, then $l_{1}<l_{2}$.

When a particular length $l$ is considered, we will define for each $s \in T_{l}$ with $\operatorname{lh}(s)=l$ a pruned tree $S_{s} \subseteq T_{l}$ such that $\left[S_{s}\right] \subseteq N_{s} \cap\left[T_{l}\right]$ and $\left[S_{s}\right]$ is clopen in $\left[T_{l}\right]$, label certain nodes in $S_{s}$ with nonempty open sets $O \subseteq \mathbb{Q}$, and take $T_{l+1}$ to be the union of all the subtrees $S_{s}$. 
To start, let $T_{0}=T$ with the root labeled with $\mathbb{Q}$. Assume a partially labeled tree $T_{l} \subseteq T$ has been defined satisfying conditions (i) through (iv). For each $s \in T_{l}$ with $\operatorname{lh}(s)=l$ define $S_{s} \subseteq T_{l}$ as follows. If $s$ is not labeled with any open set, then we do nothing: let $S_{s}$ be the unique pruned tree with $\left[S_{s}\right]=N_{s} \cap\left[T_{l}\right]$ and label no extensions of $s$ by open sets. Assume that $s$ has been labeled with a nonempty open set $O \subseteq \mathbb{Q}$. Let $t_{n} \in T_{l}, n \in \omega$, be such that $t_{n} \supseteq s$ for all $n \in \omega, N_{t_{n}} \cap N_{t_{m}}=\emptyset$ for $n \neq m$, and $N_{s} \cap\left[T_{l}\right]=\bigcup_{n}\left[N_{t_{n}}\right] \cap\left[T_{l}\right]$. Such $t_{n}$ exist since $N_{s} \cap\left[T_{l}\right]$ is clopen in $[T]$. One of the following four cases must occur:

Case 1(a). For all $n \in \omega, f\left(N_{t_{n}} \cap\left[T_{l}\right]\right)$ is nowhere dense in $O$. We have found the desired $s, t_{n} \in T$ and $O \subseteq \mathbb{Q}$, since by applying Lemma 6 a clopen subset $U \subseteq\left[T_{l}\right]$ can be found with $f\left(U \cap\left[T_{l}\right]\right)$ nonresolvable, and $U \cap\left[T_{l}\right]$ is indeed clopen in $P=[T]$.

Case 1(b). There is exactly one $k \in \omega$ for which $f\left(N_{t_{k}} \cap\left[T_{l}\right]\right)$ is somewhere dense in $O$ but not dense in $O$, and for all $n \neq k, f\left(N_{t_{n}}\right)$ is nowhere dense in $O$. Pick a nonempty open $O^{\prime} \subseteq O$ such that $f\left(N_{t_{k}}\right) \cap O^{\prime}=\emptyset$. Now each $f\left(N_{t_{n}} \cap\left[T_{l}\right]\right)$ is nowhere dense in $O^{\prime}$, while $f\left(N_{s} \cap\left[T_{l}\right]\right)$ is dense in $O^{\prime}$, so again we have found the required $s, t_{n}$ and $O^{\prime}$.

The search algorithm terminates with success in Cases 1(a) and 1(b).

Case 2. There is exactly one $k \in \omega$ such that $f\left(N_{t_{k}}\right)$ is dense in $O$, and for all $n \neq k, f\left(N_{t_{n}}\right)$ is nowhere dense in $O$. In this case let $S_{s}$ be the unique pruned tree with $\left[S_{s}\right]=N_{t_{k}} \cap\left[T_{l}\right]$ and label $t_{k}$ with $O$.

Case 3. There are at least two distinct $k_{1}, k_{2} \in \omega$ such that each of $f\left(N_{t_{k_{1}}} \cap\left[T_{l}\right]\right)$ and $f\left(N_{t_{k_{2}}} \cap\left[T_{l}\right]\right)$ is respectively dense in some nonempty open $O_{1}, O_{2} \subseteq O$. For notational simplicity, and without loss of generality, we may assume $k_{1}=1$ and $k_{2}=2$. By shrinking $O_{1}$ and $O_{2}$ if necessary, we may also assume that $O_{1}$ and $O_{2}$ are disjoint clopen sets in $\mathbb{Q}$. Now let $S_{s}$ be the unique pruned tree with

$$
\left[S_{s}\right]=\left(N_{t_{1}} \cap\left[T_{l}\right] \cap f^{-1}\left(O_{1}\right)\right) \cup\left(N_{t_{2}} \cap\left[T_{l}\right] \cap f^{-1}\left(O_{2}\right)\right) .
$$

Then $\left[S_{s}\right]$ is clopen in $\left[T_{l}\right]$ and $t_{1}, t_{2} \in S_{s}$. We then label $t_{1}$ and $t_{2}$ with $O_{1}$ and $O_{2}$, respectively.

We claim that this search algorithm always terminates in Case 1(a) or 1(b) after finitely many steps. Suppose this is not the case. Then we obtain a pruned tree $T_{\infty}=\bigcap_{l} T_{l}$ which contains all labeled nodes. If below every labeled node in $T_{\infty}$ there is a split as in Case 3, then $T_{\infty}$ has $2^{\aleph_{0}}$ many branches. Since $f\left(\left[T_{\infty}\right]\right) \subseteq \mathbb{Q}$ is countable, there are distinct branches $x \neq y \in\left[T_{\infty}\right]$ such that $f(x)=f(y)$. Let $s \subseteq x, y$ be the longest labeled node; $t_{1}$ and $t_{2}$ are labeled nodes such that $s \subsetneq t_{1} \subseteq x$ and $s \subsetneq t_{2} \subseteq y$. Then Case 3 occurs when $s$ is considered, and $t_{1}$ and $t_{2}$ are respectively labeled with disjoint clopen sets $O_{1}$ and $O_{2}$. Let $l=\operatorname{lh}(s)$. By our construction $f(x) \in f\left(N_{t_{1}} \cap\left[T_{l+1}\right]\right) \subseteq O_{1}$ and $f(y) \in f\left(N_{t_{2}} \cap\left[T_{l+1}\right]\right) \subseteq O_{2}$. Since $O_{1} \cap O_{2}=\emptyset, f(x) \neq f(y)$, a contradiction. Hence, there is an $s \in T_{\infty}$ with label $O$ such that all labeled nodes in $T_{\infty}$ extending $s$ are obtained from Case 2 . Therefore, there is $x \in\left[T_{\infty}\right]$ such that $x\lceil n$ has label $O$ for infinitely many $n \in \omega$. By our construction, $f\left(N_{x \uparrow n}\right)$ is dense in $O$ for every $n \in \omega$. On the other hand, $f$ is continuous; thus, we could pick a neighborhood $O^{\prime} \subsetneq O$ of $f(x)$ strictly smaller than $O$ and an $N_{x \uparrow n}$ such that $f\left(N_{x \uparrow n}\right) \subseteq O^{\prime}$. Then $f\left(N_{x \uparrow n}\right)$ is not dense in $O$, a contradiction. 


\section{MAIN THEOREM}

We now derive Theorem 1 from Proposition 7 . The arguments in the following proofs are essentially the same as those in 8 .

Lemma 8. Let $Y$ be a separable metrizable space and let $f: \omega^{\omega} \rightarrow Y$ be a clopenresolvable continuous surjection. Suppose $Q \subseteq Y$ is a countable perfect set and $P=f^{-1}(Q)$. Then $f \uparrow P: P \rightarrow Q$ is a clopen-resolvable continuous surjection.

Proof. Suppose $U \subseteq P$ is clopen in $P$. We need to show that $f(U)$ is resolvable in $Q$. For every $x \in U$, pick a basic clopen neighborhood $U_{x}=N_{s} \subseteq \omega^{\omega}$ for some $s \subseteq x$ with $2^{-\operatorname{lh}(s)}=\operatorname{diam} U_{x}<d(x, P \backslash U)$, where $d$ is the usual metric on $\omega^{\omega}$. Similarly, for every $x \in P \backslash U$, pick a basic clopen neighborhood $U_{x} \subseteq \omega^{\omega}$ with $\operatorname{diam} U_{x}<d(x, U)$. Finally, for $x \in \omega^{\omega} \backslash P$, pick a basic clopen neighborhood $U_{x}$ with $U_{x} \subseteq \omega^{\omega} \backslash P$. Now the collection $\left\{U_{x}: x \in \omega^{\omega}\right\}$ is an open cover of $\omega^{\omega}$ and has a countable subcover since $\omega^{\omega}$ is second countable, in particular Lindelöf. Let $U_{0}, U_{1}, \ldots$ enumerate the elements of the countable subcover. Define, for each $n \in \omega, V_{n}=U_{n}-\bigcup_{m<n} U_{m}$. Since each $U_{n}$ is clopen, we get that each $V_{n}$ is clopen. Thus $\left\{V_{n}: n \in \omega\right\}$ is an open refinement of $\left\{U_{x}: x \in \omega^{\omega}\right\}$ consisting of disjoint clopen sets such that each $V_{n}$ is a subset of some $U_{x}$. Let

$$
V=\bigcup\left\{V_{n}: V_{n} \cap U \neq \emptyset\right\} \text {. }
$$

Then $V$ is clopen in $\omega^{\omega}$ and $V \cap P=U$. Hence, $f(U)=Q \cap f(V)$. Since by assumption $f(V)$ is resolvable in $Y, f(U)$ is resolvable in $Q$.

Theorem 9. If $X$ is Polish, $Y$ is metrizable, and $f: X \rightarrow Y$ is a resolvable continuous surjection, then $Y$ is Polish.

Proof. Suppose towards a contradiction that $Y$ is not completely metrizable. By Propositions 2, 3, and Hurewicz's theorem [4, $Y$ contains a countable perfect subset $Q \subseteq Y$ homeomorphic to $\mathbb{Q}$.

Assume first that $f$ is open-resolvable. We use a classical result of Hausdorff [2] that there is a continuous open surjection $g: \omega^{\omega} \rightarrow X$. The composition $f \circ g$ is now clopen-resolvable, and by Lemma 8 so is $f \circ g \uparrow P: P \rightarrow Q$, where $P=$ $(f \circ g)^{-1}(Q)$. This contradicts Proposition 7 . If $f$ is closed-resolvable, we use a continuous closed surjection $g: \omega^{\omega} \rightarrow X$ given by the theorem of Engelking [1] (cf. proof of Proposition (3) and obtain a contradiction in a similar fashion.

\section{REFERENCES}

[1] R. Engelking, On closed images of the space of irrationals. Proc. Amer. Math. Soc. 21 (3) (1969), 583-586. MR0239571 (39:928)

[2] F. Hausdorff, Über innere Abbildungen, Fund. Math. 23 (1934), 279-291.

[3] P. Holický and R. Pol, On a question by Alexey Ostrovsky concerning preservation of completeness, to appear in Topology Appl.

[4] W. Hurewicz, Relativ perfekte Teile von Punktmengen und Mengen (A), Fund. Math. 12 (1928), 78-109.

[5] A. S. Kechris, Classical descriptive set theory. Graduate Texts in Mathematics, 156, Springer, 1995. MR1321597 (96e:03057)

[6] K. Kuratowski, Topology, volume 1, Academic Press, New York, 1966.

[7] E. Michael and A. H. Stone, Quotients of the space of irrationals. Pacific J. Math. 28 (3) (1969), 629-633. MR0256346(41:1002)

[8] A. Ostrovsky, New basic result in classical descriptive set theory: Preservation of completeness. Topology Appl. 156 (2009), 1749-1751. MR2521712 
[9] W. Sierpiński, Sur un propriete des ensembles $G_{\delta}$, Fund. Math. 16 (1930), 173-180.

[10] I. A. Vainštain, On closed mappings. Moskov. Gos. Univ. Uč. Zap. 155 (5) (1952), 3-53 (in Russian). MR0077113(17:992b)

Department of Mathematics, University of North Texas, 1155 Union Circle \#311430, Denton, Texas 76203-5017

E-mail address: sgao@unt.edu

Department of Mathematics, University of North Texas, 1155 Union Circle \#311430, Denton, Texas 76203-5017

E-mail address: kieftenbeld@unt.edu 